\title{
0735-1933(95)00084-4
}

\section{AN IMPROVED MODEL FOR THE SINGLE-BLOW MEASUREMENT INCLUDING THE NON-ADIABATIC SIDE WALL EFFECT}

\author{
Ping-Hei Chen and Zei-Chi Chang \\ National Taiwan University \\ Department of Mechanical Engineering \\ Taipei, Taiwan \\ Republic of China
}

(Communicated by J.P. Hartnett and W.J. Minkowycz)

\begin{abstract}
The present study proposes an improved model for the single-blow technique which is commonly used to measure the thermal performance of a cryocooler's regenerator matrix. The improvements in the present model include the heat flux from the fluid into the side wall and the axial conduction of the wire-screen matrix and side wall as a suddenly heated fluid passes through the wire-screen matrix. Numerical results obtained from the present model are compared with both the analytic results that exclude the axial conduction and the predicted results of a prior study that neglect the heat flux into the side wall. Results are also presented to illustrate the effect of the heat capacity of the side wall, the heat flux into the side wall, and the axial conduction on both the exit fluid temperature response and the $N T U$ value.
\end{abstract}

\section{$\underline{\text { Introduction }}$}

A transient single-blow technique has been commonly used for determining the $N T U$ value of a compact heat exchanger [1-6] and the regenerator of cryocooler [7-8]. The single-blow technique requires a steady fluid flow passing through the test heat exchanger. In addition, both the fluid flow and test heat exchanger are in thermal equilibrium before the sudden change in the inlet fluid temperature. Once the inlet fluid temperature is suddenly heated up or cooled down, the 
heat transfer starts to take place between the fluid flow and the heat exchanger as well as the side wall. Using the inlet temperature response as an initial condition, the exit fluid temperature response history of the test heat exchanger can be estimated from an adequate model.

Since a unique relationship exists between the $N T U$ value of the heat exchanger and the exit fluid temperature response, the $N T U$ value of the test heat exchanger is then evaluated by matching the recorded measured exit fluid temperature history with the predicted one. Details of the procedure for determining the $N T U$ value and the choice of scheme for matching experimental and predicted data were reported by Liang and Yang [3]. Their proposed five-point matching scheme was also used by Cai et al. [1]. In addition the curve-matching scheme, a maximum-slope scheme [2] is also frequently used to evaluate the $N T U$ value of the test heat exchanger since a unique relation also exists between the $N T U$ value and the maximum-slope value of the exit fluid temperature response. It is worth noting that the determined $N T U$ value of the test heat exchanger varies with the model used in the single-blow measurement. It is due to the fact that different exit fluid temperature response curves are obtained from different models but the measured one being matched is unchanged. For instance, the $N T U$ value of the test heat exchanger, obtained from the model including the axial conduction, is significantly higher than the one obtained from the model without considering the axial conduction for $\lambda \times N T U>0.06$ [1].

Despite the numerous studies [1-8] that have been conducted, none of the previous studies reported their measured $N T U$ values greater than 80 of the test heat exchanger or wire-screen matrix. However, a cryocooler's regenerator which is stacked with hundreds of oversized wire screens onto a circular tube can have a large $N T U$ value. Additional attention should be paid to the model of the single-blow measurements for the regenerator matrix due to its large $N T U$ value.

To our knowledge, all prior studies considered the side wall as adiabatic. For a test this assumption may cause a serious error as a heat exchanger or wire-screen matrix is tightly fit with the rectangular or circular side wall, or for a single-blow measurement requiring a long period of operation. Therefore, the aim of the present study is to propose an improved model for the singleblow technique as the side wall cannot be reasonably assumed to be adiabatic.

\section{The Improved Model for the Single-Blow Method}

The prior models for the single-blow test usually regard the side wall as adiabatic and fluid flow as one-dimensional. Therefore, there are only two unknowns, namely fluid temperature $T_{f}^{*}$ 
and the temperature of the test regenerator matrix $T^{*}$, in such a single-blow model. However, if the heat transfer also occurs between the side wall and fluid flow, an additional unknown appears: the side wall temperature $T^{*}$. If a one-dimensional fluid flow with constant properties is assumed to pass through the test regenerator matrix, the present improved model require three governing equations to describe the single-blow system, as shown in Fig. 1, for solving three unknowns, $T_{f}^{*}$, $T_{s}^{*}$, and $T^{*}$.

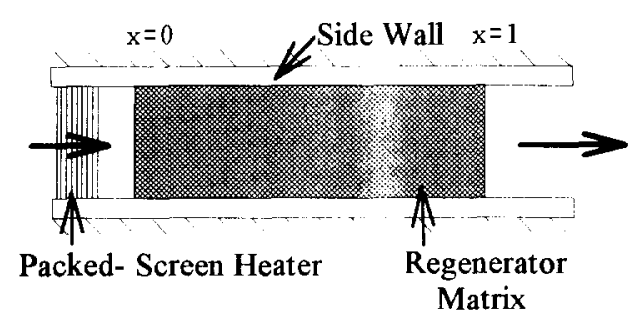

FIG. 1

Schematic View of a Single-Blow Measurement

From the energy balance between the fluid flow and the test regenerator matrix as well as the side wall, it yields the dimensionless energy equations for the fluid,

$$
\frac{\partial T_{f}}{\partial x}+\operatorname{NTU}\left(T_{f}-T_{s}\right)+N T U_{n}\left(T_{f}-T_{n}\right)=0
$$

for the regenerator matrix,

$$
\frac{\partial T_{f}}{\partial t}-\lambda \frac{\partial^{2} T_{s}}{\partial x^{2}}+N T U\left(T_{s}-T_{f}\right)=0
$$

and for the side wall,

$$
\frac{\partial T_{w}}{\partial t}-R_{t c} \cdot \lambda_{n} \frac{\partial^{2} T_{w}}{\partial x^{2}}+R_{r c} \cdot N T U_{n}\left(T_{n}-T_{f}\right)=0
$$

The dimensionless variables or parameters in Eqns (1)-(3) are defined as follows:

$$
T_{f}=\frac{T_{f}^{*}-T_{0}^{*}}{T_{f \max }^{*}-T_{0}^{*}}, T_{s}=\frac{T_{s}^{*}-T_{0}^{*}}{T_{f \max }^{*}-T_{0}^{*}}, T_{w}=\frac{T_{\mu}^{*}-T_{0}^{*}}{T_{f \max }^{*}-T_{0}^{*}}, x=\frac{x^{*}}{L},
$$




$$
\begin{gathered}
t=\frac{t^{*}}{\left(M_{s} C_{s} / \dot{m}_{f} C_{P}\right)}, R_{t}=\frac{M_{s} C_{s}}{M_{s} C_{s}} \\
N T U=\frac{h A_{H T}}{\dot{m}_{f} C_{P}}, N T U_{w}=\frac{h_{w} A_{H T}}{\dot{m}_{f} C_{P}}, \lambda=\frac{k_{t f f e r} A_{t}}{m_{f} C_{p} L} \text { and } \lambda_{w}=\frac{k_{w} A_{w}}{\dot{m}_{f} C_{p} L}
\end{gathered}
$$

As compared with the model $[1,2]$ with an adiabatic side wall, additional nondimensional groups such as $N T U_{w}, R_{t c}$ and $\lambda_{w}$ are included in the present improved model. Eqns (1)-(3) are subject to the following initial and boundary conditions

$$
\begin{aligned}
& t=0, T_{f}(x)=T_{s}(x)=T_{n}(x)=0
\end{aligned}
$$

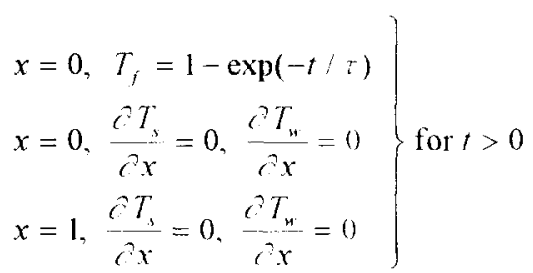

where $\tau$ is the nondimensional time constant of the suddenly heated inlet fluid temperature response

If the values of $h$ and $h_{w}$ are assumed to be the same, the procedure for evaluating the $N T U$ value matrix can be stated as follows. The first step) is to guess the value of $h$. Next, the exit fluid temperature history is obtained by solving Eqns (1)-(3). This predicted fluid temperature history is then compared with the measured one. If both measured and predicted exit fluid temperature histories lie within a certain limit, the guessed $h$ value corresponds with the averaged heat transfer coefficient of the regenerator matrix. Otherwise. the procedure is repeated until the $h$ value is found.

\section{Analytical Solution}

An analytical solution of Eqns (1)-(3) can be obtained by using the Laplace transform if the conduction terms are neglected. The detailed process not presented in the paper is very tedious, and the solution is expressed as 


$$
\begin{aligned}
& T_{f}(t, x=1)=\frac{e^{-\left(N T U+N T U_{w}\right)}}{\tau} \cdot\left\{\int_{0}^{t} e^{-N T U /(t-z)} I_{11}\left[2\left(P_{1}(t-z)\right)^{1 / 2}\right] \cdot e^{-P_{1} z} I_{0}\left[2\left(P_{2} z\right)^{1 / 2}\right] d z\right. \\
& +\int_{0}^{t} e^{-N T U(t-z)} I_{0}\left[2\left(P_{1}(t-z)\right)^{1 / 2}\right] \cdot \int_{11}^{t} e^{-l_{l^{\prime \prime}}^{\prime \prime}} I_{0}\left[2\left(P_{2} \eta\right)^{1 / 2}\right] d \eta d z \\
& +\int_{0}^{t} e^{-P_{1}(t-z)} I_{0}\left[2\left(P_{2}(t-z)\right)^{1 / 2}\right] \cdot \int_{0}^{l} e^{-N\left(t u{ }^{\prime}\right.} I_{1)}\left[2\left(P_{1} \eta\right)^{1 / 2}\right] d \eta d z \\
& +\int_{0}^{t} \int_{0}^{t-z} e^{-N T U \cdot \eta} I_{0}\left[2\left(P_{1} \eta\right)^{1 / 2}\right] d \eta \cdot \int_{11}^{2} e^{-P_{1} \zeta} I_{0}\left[2\left(P_{2} \xi\right)^{1 / 2}\right] d \xi d z \\
& +\int_{0}^{t} \frac{e^{-(t-\xi) / \tau}}{\tau} \int_{0}^{\xi} e^{-v T U / 4-(\xi-z)} I_{0}\left[2\left(P_{1}(\xi-z)\right)^{1 / 2} \cdot e^{-P_{1} z} I_{0}\left[2\left(P_{2} z\right)^{1 / 2}\right] d z d \xi\right. \\
& +\int_{0}^{t} \frac{e^{-(t-5) / \tau}}{\tau} \int_{0}^{\xi} e^{-N T I(t--z)} I_{0}\left[2\left(P_{1}(\xi-z)\right)^{1 / 2}\right] \cdot \int_{0}^{z} e^{-P_{1 \eta} \eta} I_{00}\left[2\left(P_{2} \eta\right)^{1 / 2}\right] d \eta d z d \xi \\
& +\int_{0}^{t} \frac{e^{-(t-\xi)} \tau}{\tau} \int_{0}^{\xi} e^{-P_{3}(\xi-z)} I_{0}\left[2\left(P_{2}(\xi-z)\right)^{1 / 2}\right] \cdot \int_{0}^{z} e^{-k T i \eta_{1}} I_{0}\left[2\left(P_{1} \eta\right)^{1 / 2}\right] d \eta d z d \xi \\
& \left.+\int_{0}^{t} \frac{e^{-(t-\xi)} \tau}{\tau} \int_{0}^{\xi} \int_{0}^{\xi-\eta \eta} e^{-N T I \cdot \eta} I_{0}\left[2\left(I_{1} \eta\right)^{1 / 2}\right] d \eta \cdot \int_{01}^{z} e^{-P_{3} \sigma} I_{0}\left[2\left(P_{2} \sigma\right)^{1 / 2}\right] d \sigma d z d \xi\right\}
\end{aligned}
$$

where $P_{1}=N T U^{2} x, P_{2}=R_{t e} N T U_{n}{ }^{2} x$, and $P_{3}=R_{t i} N T U_{n}$.

However, the analytic solution cannot be obtained for the arbitrary inlet temperature rise in the single-blow method. Nevertheless, the analytic solution can still serve as a baseline check for the solution obtained from the finite-difference method.

\section{Numerical Solutions by a Finite-Difference Scheme}

As the axial conduction is considered, Eqns (1)-(3) can only be solved numerically. If the temperature distributions of $T_{f}, T_{s}$, and $T_{w}$ at the ith time step are known, then the values of $T_{s}$ and $T_{w}$ at the $(i+1)$ th time step can be easily obtained from the discretized form of Eqns (2) and (3) by an explicit finite-difference scheme. Once the values of $T_{s}^{i} /$ and $T_{w}^{i+1}$ are obtained, Eq.(1) is then discretized to solve $T_{f}^{i+1}$ using a central difference scheme which has a second-order accurate in space.

To improve the time accuracy of numerical solutions, the predicted values of $T_{s}$ and $T_{w}$ at the $(i+1)$ th time step are further corrected by a Crank-Nicolson scheme. The new value of $T_{f}$ at $(i+1)$ th time step is again obtained by substituting the corrected $T_{v}^{i-1}$ and $T_{w}{ }^{i+l}$ into the discertized form of Eq.(1) 


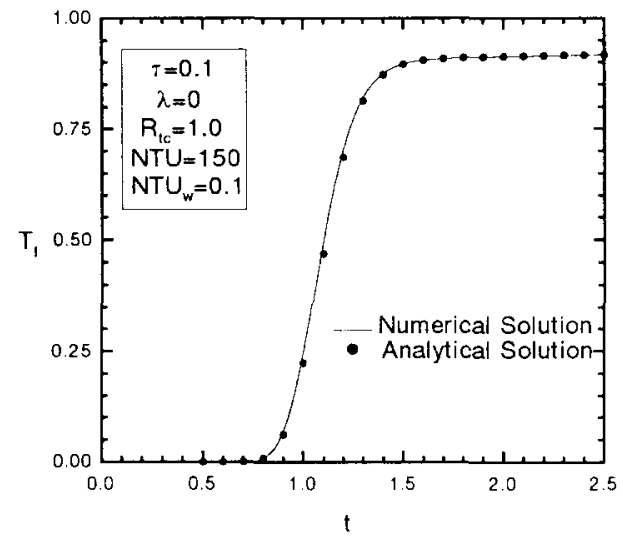

FIG. 2

Comparison Between Analytic and Numerical Predictions of $T_{f}$ at $x=1$

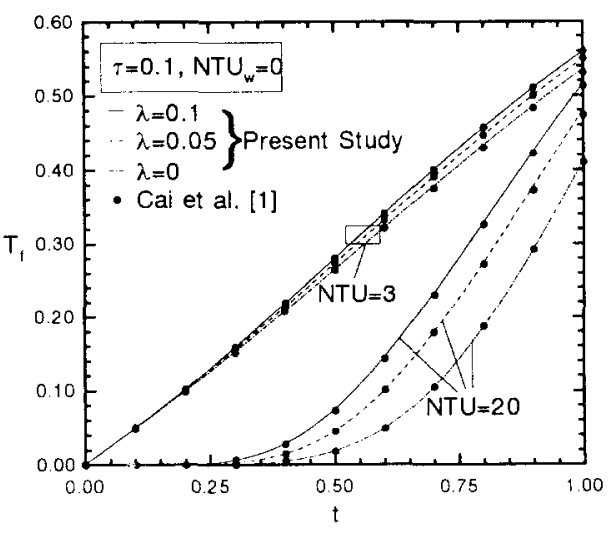

FIG. 3

Comparison Between the present predicted $T_{f}$ value at $x=1$ and those in Cai et al.[1]

For the case with $\tau=0.1, N T U=150, N T U_{w}-0.1$, and $R_{t c}=1$, the exit fluid temperature response curves determined from both the exact analytical and numerical solutions show an excellent agreement with each other in Fig. 2. If the axial conduction is included, the numerical predictions are also compared with the predicted results in Cai et al. [1] at various values of $\lambda$ with the adiabatic side wall, as shown in Fig. 3. This figure also demonstrates a good agreement between both results.

\section{Test Conditions}

For a regenerator in a typical cryocooler. the values of $N T U, \lambda, N T U_{w}$, and $R_{t c}$ are approximately around 60 to $150,0.0001$ to 0.005 .0 .01 to 0.001 , and 1 to 10 , respectively. The axial conduction of the side wall $\lambda_{n}$ is neglected due to its insignificant effect on the exit fluid temperature response for small $N T U_{w}$ values. If the fluid flow is heated using a packed-screen 
heater in the single-blow test, the $\tau$ value of the inlet temperature rise is about 0.1 . The value of parameters in the aforementioned ranges will be used for predicting the exit fluid temperature responses.

\section{$\underline{\text { Results and Discussions }}$}

Numerical predictions are presented to show the effects of some important factors included in the present improved model for the transient single-blow method on both the exit fluid temperature response and the evaluation of the $N T U$ value of the test regenerator matrix. Those factors of particular interest are the heat flux into side wall, the heat capacity of the side wall, and the axial conduction. In the first part, results are presented to show the effect of these factors on the exit fluid temperature response.

\section{Exit Fluid Temperature Responses}

For a typical regenerator matrix, the ratio of $N T U_{w}$ to $N T U$ is usually very small because the heat transfer area for the wire-screen matrix is comparably larger than that for the side wall. Since the ratio is small, its effect on the exit fluid temperature response has not been assessed by prior studies. Unfortunately, it may cause some errors in determining the $N T U$ value of the regenerator matrix even though its value is small. To assess its effect, another parameter $R_{N T U}$ is introduced and given by

$$
R_{N T I:}=\frac{N T U_{u}}{N T U}
$$

The present study makes an assumption that the $R_{\vee}$ value is the same as the heat transfer area ratio of the side wall to the regenerator matrix. In the following sections, the parameter $R_{N T L}$ is used to assess the effect of heat flow into the side wall rather than the parameter $N T U_{w}$.

If the inlet fluid temperature is an exponential rise as stated as in Eq.(6), Fig. 4 illustrates the numerical results at $\tau=0.1$ and $N T U=60$ for $R_{t c}$ ranging from 1 to 10 and $R_{N T C}$ ranging from 0.0 to 0.01 . For the test regenerator with a small value of $R_{i \eta n}$. say $R_{N T L}=0.001$, it can be observed that there is little effect on the initial rise of the exit fluid temperature for $t<1$ with the variation in 
the $R_{t c}$ value. For $t<1$, most thermal energy is transferred from the heated fluid flow into the matrix rather than into the side wall because the heat transfer area of the regenerator matrix is one thousand times as large as the heat that of the side wall. For the case at $R_{N 77}=0.001$, notable differences in the exit fluid temperature response only occur for $t>1.6$. This is because the regenerator matrix has already reached the thermal equilibrium state with the fluid flow for the large $t$ value. Therefore, the heat transfer only takes place between the side wall and the heated fluid flow. Note that the $T_{f}$ value is still about $5 \%$ lower than $T_{\text {max }}$ at $R_{\mathrm{fc}}=1$ and $R_{\text {NTl }}=0.001$ for $2.5>t>1.6$. Therefore, one should be very careful in making an assumption for the adiabatic side wall in the single-blow method. For the test regenerator matrix with a large $N T U$ value, such an assumption may cause some errors in evaluating the $N T U$ value of the test piece.

While the $R_{N T V}$ value rises from 0.001 to 0.01 . the influence of the $R_{c c}$ value on the exit fluid temperature becomes more significant. For the case of $N T U=60$ and $R_{N T}=0.01$, the variation in the exit fluid temperature is large as the $R_{t}$ value drops from 10 to 1 for $t>1.0$. However, the exit fluid temperature at a larger $R_{V T T}$ : value reaches the $T_{\text {max }}$ more quickly than that at a smaller $R_{N T U}$ value due to a larger heat flux from the lieated working fluid into the side wall.

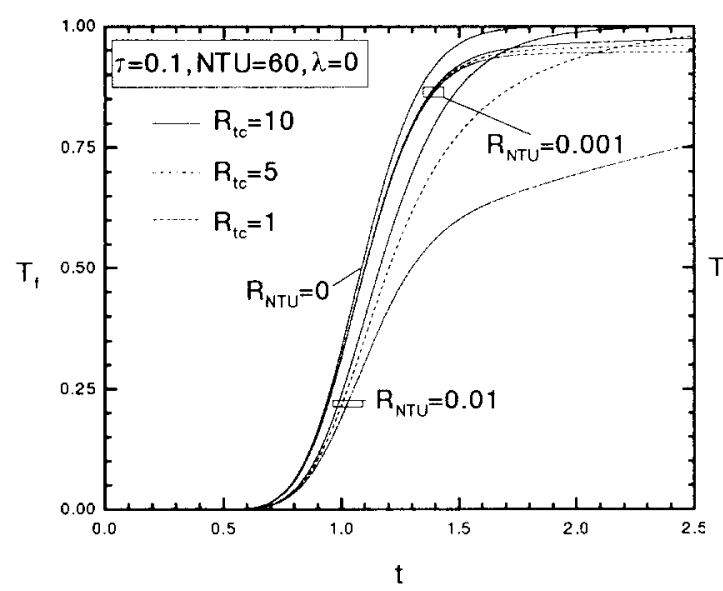

FIG. 4

Exit Fluid Temperature Response with the Non-Adiabatic Side Wall

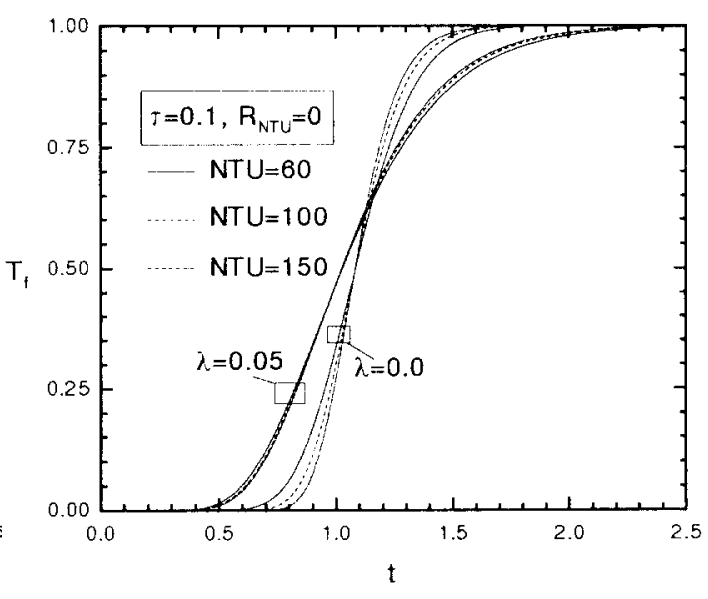

FIG. 5

Exit Fluid Temperature Responses

at Various $N T U$ Values with $R_{N 7 U}=0$ 
With an adiabatic side wall, Fig. 5 shows the exit fluid temperature response curves for various values of NTU and $\lambda$ at $\tau=0.1$ and $R_{c c}=10$. Predicted results illustrate that the exit fluid temperature response curves become difficult to distinguish as the $N T U$ value increases. Unfortunately, the exit fluid temperature response curves at different $N T U$ values are even closer once the axial conduction in the test regenerator is included in the single-blow model. In addition, the exit fluid temperature response is also significantly varied with the axial conduction of the test regenerator matrix. When the axial conduction is considered. the temperature difference between the inlet and outlet fluid temperature becomes smaller with an increase in the $\lambda$ value for a small value of $t$. As a result, a sharper temperature rise is observed for the case with a larger $\lambda$ value. Consequently, the $N T U$ value of the regenerator matrix, determined by the maximum-slope method, would be underestimated if axial conduction is ignored in the model for predicting the exit fluid temperature response. Note that the predicted results are presented for the case with the adiabatic side-wall. Otherwise, the underestimation of the $N T U /$ value is more serious once the heat flow into the side wall is also excluded in the single-blow model.

\section{Effect of Axial Conduction and Non-Adiabatic Side Wall on the NTU value}

The aim of the single-blow method is to determine the $N T U$ value of a test regenerator matrix by matching both measured and predicted exit fluid temperature response for a known inlet fluid temperature rise. Therefore, it is necessary to provide the correct predictions for a match with the experimental data. The aforementioned parameters which can significantly affect the exit fluid temperature response, accordingly, should not be exclucled in the single-blow method for the test regenerator matrix with large $N T U$ value. To determine the $N T U$ value of the regenerator matrix, two popular schemes, the curve-matching scheme [1.3] and the maximum-slope scheme [2], have been employed in the single-blow method. Nevertheless. the maximum slope technique is chosen in the present analysis.

As mentioned earlier, the $N T U$ value would be underestimated if the exit fluid temperature response at $\lambda=0$ is used to match that with a finite $\lambda$ value. For comparison, the underestimated $N T U$ value of the test regenerator matrix at $\lambda=0.01$ is illustrated in Fig. 6 for both the curvematching in Cai et al. [1] and the maximum-slope scleme employed in the present study. The agreement is quite consistent between both studies. However, the deviation between both schemes 
increases with the $\lambda$ value. Note that the same and correct $N T U$ value of the test regenerator matrix should be determined if both schemes all consiler the actual axial conduction effect in the single-blow method. Therefore, the data in Fig. 6 only shows that the underestimated value in $N T U$ if the exit fluid temperature response with $\lambda=0$ is used to match that with $\lambda \neq 0$ at a particular $N T U$ value. From Fig. 6 , the underestimation in $N T U$ with $\lambda=0$ using the maximum-slope scheme is less severe than using the curve-matching scheme. For example, according to Fig. 6 , an exit temperature response at $N T U=20$ and $\lambda=0.03$ has the best match with that at $N T U=13.8$ and $\lambda$ $=0$ using the curve-matching scheme. However, the maximum-slope value of the exit fluid temperature response at $N T U=15.7$ at $\lambda=0$ is same as that at $N T U=20$ and $\lambda=0.03$. Therefore, the maximum-slope method is preferred if the model in the single-blow method neglects the axial conduction in the test heat exchanger for $N T U \cdot 8$. Furthermore, in the curve matching technique, the determined $N T U$ value varies with the selection of data points in the exit fluid temperature to be matched with the experimental data. Therefore, Cai et al [1] proposed a method to determine the optimum matching time beyond which five equally-interval points were selected for matching.

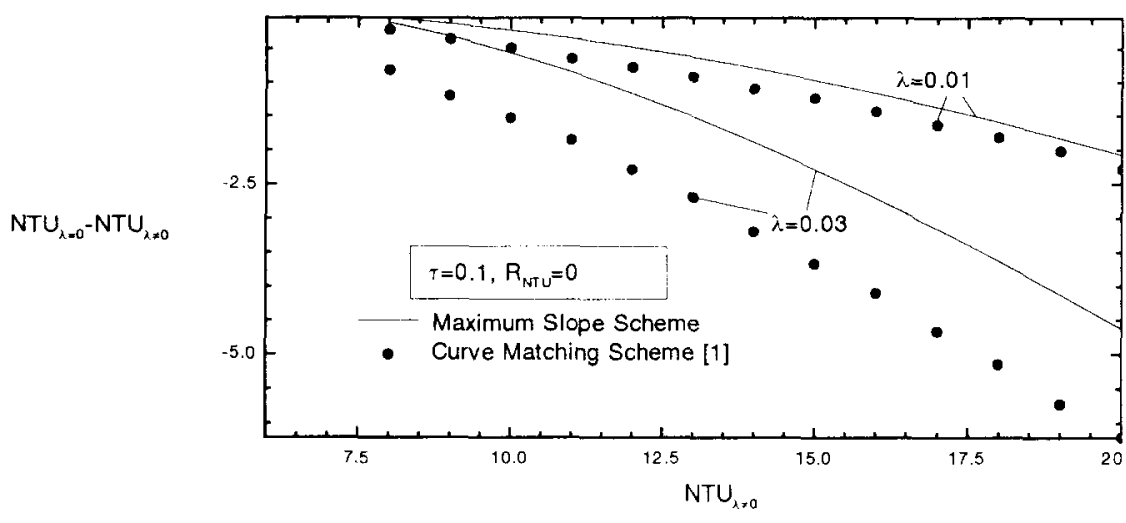

FICi. 6

Effect of the Axial Conduction on the Evaluation of $N T U$ Value by Both the Maximum Slope Scheme and the Curve Matching Scheme in Cai et al. [1]

On the contrary, a unique relation exists between the NTU/ value and the maximum-slope of the exit fluid temperature response. In the maximum-slope scheme, there is no need to find the optimum matching time and to decide how many points are chosen for matching. Based on these findings, the maximum slope technique is employed in the following analysis for the effect of $\lambda$ and 
$R_{N T U}$ values on the determination of $N T U$ value,

For a typical regenerator, the $N T U$ value may be over 100 due to its large heat transfer area. Meanwhile, the effective thermal conductivity of a wire-screen matrix is much less than the material conductivity due to the small thermal contact conductance between wire screens. For a stainless steel wire-screen with 100 mesh, the effective thermal conductivity $k_{e f f e c t}$ is around $0.07 \mathrm{~W} / \mathrm{m}^{\circ} \mathrm{C}$ as reported by Chang [9]. As a result, the dimensionless $\lambda$ value for a regenerator matrix is usually less than 0.005 . Fig. 7 shows the effects of the $\lambda$ and $R_{N T T}$ values on the maximum slope of the exit fluid temperature in a wide range of $N T U$ for $\tau=0.1, R_{t c}=10.0$. As the $N T U$ value of the test regenerator matrix is less than 10 , it is reasonable to make the assumptions for the adiabatic side-wall and no axial conduction in the test heat exchanger for the range $\lambda$ and $R_{N T U}$ being studied. With an increase in the $N T U$ value. both effects of $\lambda$ and $R_{N T U}$ on the maximumslope value become more significant. If the maximum-slope value of the exit fluid temperature response is obtained from the experimental data, the $N T U /$ value of the test regenerator matrix is underestimated by matching the predicted one from the model which excludes the effect of both the heat flux into the side-wall and the axial conduction in the regenerator matrix.

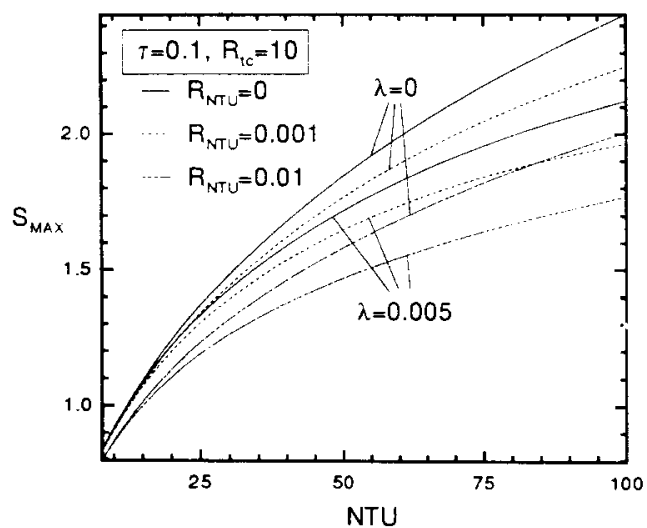

FIG. 7

Maximum Slope of Exit Fluid Temperature Response for Various $R_{N T /}$ and $\lambda$ Values

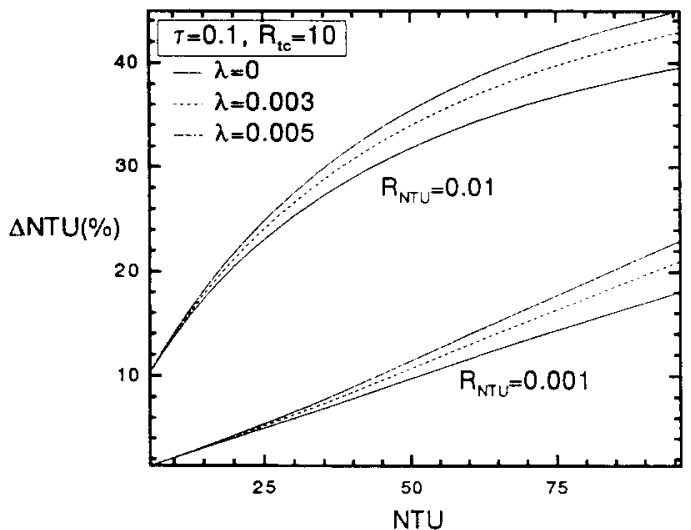

FIG. 8

Effect of the Non-Adiabatic Side Wall on the Estimated $N T U$ Value for Various $\lambda$ Values 
Based on the the same $S_{\text {mav }}$ values in Fig. 7. the variation percentage in the predicted $N T U$ value with and without including both $\lambda$ and $R_{\text {, }}$ values is plotted in Fig. 8. The parameter $A N T U$ on the vertical axis is given by

$$
\operatorname{SNTU}(\%)=\frac{N T U-N T U_{R_{N 2},-11, \ldots 1}}{N T U} \times 100
$$

where the $N T U$ value is determined from the model with the consideration of the non-adiabatic side wall at various $\lambda$ values. Fig. 8 demonstrates the valiation percentage increases with both $\lambda$ and $R_{N T L}$. As observed in Fig. 8, both effects of $R_{\checkmark /:}$ and $i$ should be considered on the evaluation of the $N T U$ value of the regenerator matrix for $N T l_{"} \quad 0.08$ or $\lambda \times N T U>0.3$

\section{Conclusions}

The improved model for the single-blow method is proposed to include the non-adiabatic side wall and the axial conduction for evaluating the NTL value of the test regenerator matrix. Numerical predictions of the exit fluid temperature response for the improved model are presented. For comparison, the analytical solutions for the model without the axial conduction are also illustrated to show the accuracy of the numerical predictions. For the test regenerator with a large $N T U$ value, the maximum-slope scheme is a better one than the curve-matching scheme for matching the experimental and numerical data. There is no need for the maximum-slope scheme to choose the time interval in which the data points are used in order to be matched with the experimental data. As the maximum-slope scheme is cmployed. the presented results also show that the $N T U$ value of the test regenerator matrix with the adiabatic side-wall is much lower than that with the non-adiabatic side wall in the single-blon model for the regenerator matrix with a large $N T U$ value. Therefore, the NTU value of the lest regenerator matrix is seriously underestimated if the adiabatic side-wall is assumed in the single-blow model. In the range of parameters being conducted in the present study. the underestimated percentage in the evaluation of the $N T U$ value of the regenerator matrix is greater than $10^{\circ}$ of for $N T U_{w}>0.08$. The error for underestimating the $N T U$ value increases with $.7 T, \lambda$ ind $R, 1$. 


\section{Nomenclature}

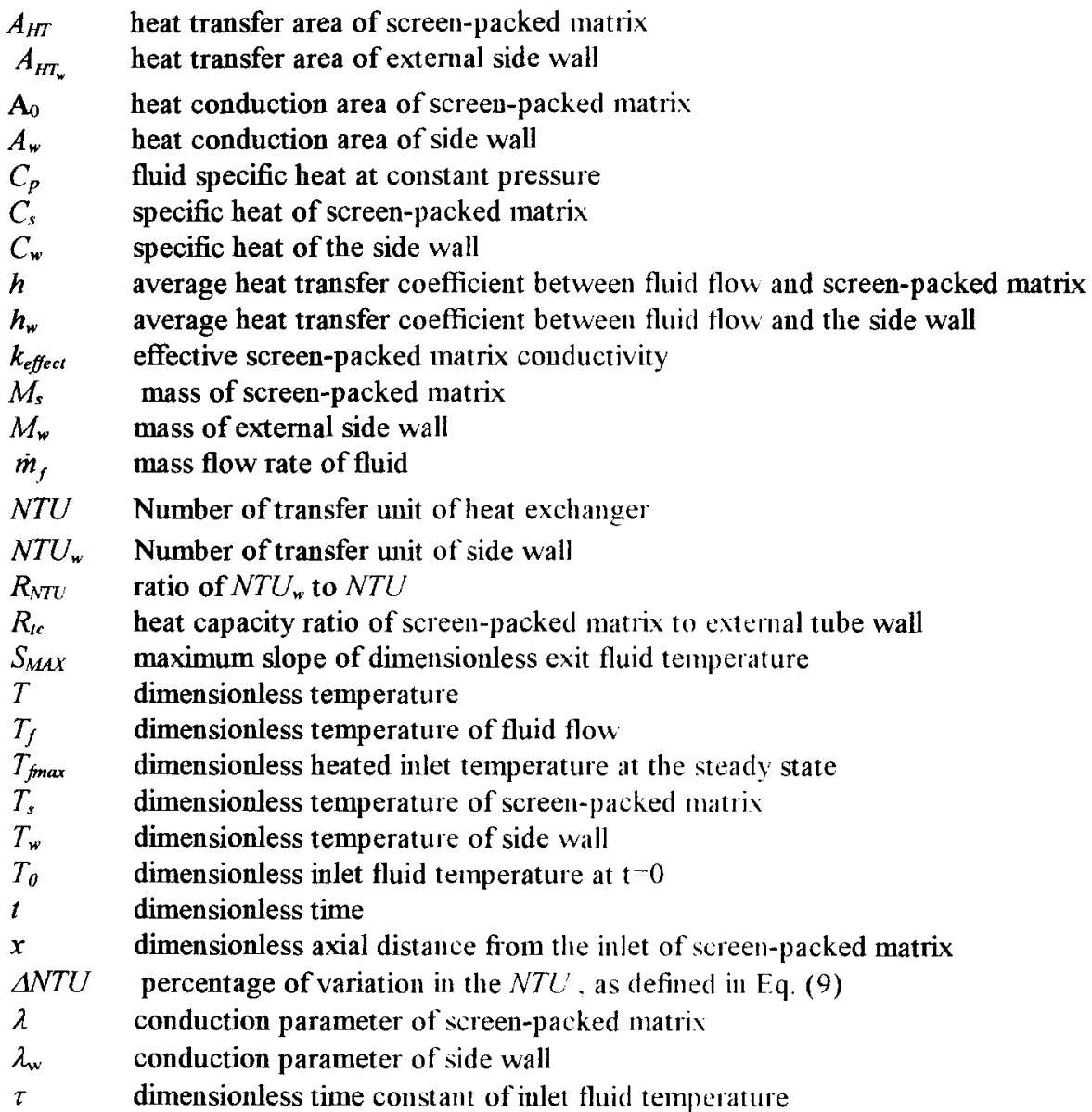

\section{Superscripts}

$i \quad$ node index in time

* dimensional variable $\underline{\text { Subscripts }}$

$\begin{array}{ll}f & \text { fluid } \\ w & \text { side wall }\end{array}$




\section{References}

1. Z.H. Cai, M.L. Li, Y.W. Wu and H.S. Ren. Imt. J. Heat Atass Transfer, 27, 971 (1984).

2. C.P. Howard, ASME Paper No.6+-GT2-11. ( 1964$)$

3. C.Y. Liang and W.J. Yang, ASME J. of Heat Transfor, 97. 16 (1975).

4. R.S. Mullisen, and R.I. Loehrke, ASME J. of Heat Trams/er. 108, 370 (1986).

5. P.F. Pucci, C.P. Howard and C.II. Piersall, ASME J. of Lngineering for Power, 96, 81 (1967).

6. Y. Yagi and S. Mochizuki, Heat Transfer-Japamese Re'se'rnch, 20. 646 (1991).

7. J.C. Kim and E.B. Qvale, Advances in Cryogenic Engine'ring, 15, 302 (1970).

8. K. Hamaguchi, S. Takahachi and H. Miyabe, JSME Series B (in Japanese), 49 , 2001 (1983).

9. W.S. Chang, ASME J. of Heat Transfer, 112, 5(1990). 